\title{
Homotopy dominations within polyhedra
}

\author{
by \\ Danuta Kołodziejczyk (Warszawa)
}

\begin{abstract}
We show the existence of a finite polyhedron $P$ dominating infinitely many different homotopy types of finite polyhedra and such that there is a bound on the lengths of all strictly descending sequences of homotopy types dominated by $P$. This answers a question of K. Borsuk (1979) dealing with shape-theoretic notions of "capacity" and "depth" of compact metric spaces. Moreover, $\pi_{1}(P)$ may be any given non-abelian poly-ZZ-group and $\operatorname{dim} P$ may be any given integer $n \geq 3$.
\end{abstract}

1. Introduction. In the paper, every polyhedron is assumed to be finite and connected.

Following K. Borsuk, define the capacity $C(A)$ of a compactum $A$ as the cardinality of the class of shapes of all compacta $X$ such that $\operatorname{Sh}(X) \leq$ $\operatorname{Sh}(A)$. (The basic notions and facts of shape theory can be found in [B2], [DS], [MS].)

A system consisting of $k$ compacta $X_{1}, \ldots, X_{k}$ is said to be a chain of length $k$ for a compactum $A$ if $\operatorname{Sh}\left(X_{1}\right)<\ldots<\operatorname{Sh}\left(X_{k}\right) \leq \operatorname{Sh}(A)(\operatorname{Sh}(X)<$ $\operatorname{Sh}(Y)$ if and only if $\operatorname{Sh}(X) \leq \operatorname{Sh}(Y)$ holds but $\operatorname{Sh}(Y) \leq \operatorname{Sh}(X)$ fails).

The depth $D(A)$ of a compactum $A$ is the least upper bound of the lengths of all chains for $A$. If this upper bound is infinite, we write $D(A)=\aleph_{0}$.

Let us remark that on ANRs shape theory coincides with homotopy theory. Moreover, in the case where $A$ is a polyhedron, one may replace the above definitions by their "homotopy versions" (i.e. in the homotopy category of $C W$-complexes). Indeed, by the classical results in shape theory (see [HaHe1] or [HaHe2]; [DS, Theorem 2.2.6]; [EG]) there is a 1-1 functorial correspondence between the shapes of compacta shape dominated by a given polyhedron and the homotopy types of $C W$-complexes (not necessar-

2000 Mathematics Subject Classification: 55P55, 55P15.

Key words and phrases: polyhedron, $C W$-complex, homotopy domination, homotopy type, compactum, shape domination, shape, capacity, depth.

The author would like to thank the Institute of Mathematics of the Polish Academy of Sciences for its support while part of this work was done. 
ily finite) homotopy dominated by that polyhedron (in both pointed and unpointed cases). Thus, if $X$ and $Y$ are two compacta shape dominated by a polyhedron, and $X^{\prime}$ and $Y^{\prime}$ are the corresponding $C W$-complexes, then $\operatorname{Sh}(X) \leq \operatorname{Sh}(Y)$ if and only if $X^{\prime} \leq Y^{\prime}$ (where $\leq$ denotes homotopy domination). Furthermore, it is immaterial whether we consider the pointed or unpointed case (by $[D]$ ).

The capacity and depth were introduced by K. Borsuk at the Topological Conference in Moscow in 1979 together with some relevant problems (see [B1]). The answers to most of them were given in [K1] (see also [K]). In [K5], answering the interesting question (4) of [B1]: Is the capacity of each polyhedron finite?, we showed that there is a polyhedron dominating infinitely many different homotopy types of polyhedra (see also [K6]).

In another formulation K. Borsuk stated this problem in 1968, at the Topological Conference in Herceg-Novi.

It was known earlier (by the results of $[\mathrm{M}],[\mathrm{Ho}]$, see also $[\mathrm{MP}]$ ) that every polyhedron dominates no more than $\aleph_{0}$ different homotopy types (or shapes). However, it was rather unexpected that there exists a polyhedron dominating infinitely many different homotopy types of polyhedra.

In this paper we show that such examples are even frequent: for every non-abelian poly-ZZ-group $G$ and integer $n \geq 3$, there exists a polyhedron with this property with fundamental group $G$ and of dimension $n$. Therefore there exist polyhedra with nilpotent fundamental groups dominating infinitely many different homotopy types (or shapes). On the other hand, in [K4] we proved that every simply-connected polyhedron dominates only finitely many different homotopy types (or shapes). Recently, we have shown that the capacity of each polyhedron with finite fundamental group and of each nilpotent polyhedron is also finite (see [K3]). Thus Borsuk's question remains unsettled only for polyhedra with abelian fundamental groups.

Using the above results, we obtain an affirmative answer to the following question of K. Borsuk (question (8) of [B1], see also [DKN, Problem (6.1)]):

Is there a compactum with infinite capacity and finite depth?

2. Algebraic preliminaries. Let us recall some definitions (see $[\mathrm{R}$, Ch. 5]). Given a group $G$ we define the lower central series of $G$,

$$
G=G_{(0)} \supseteq G_{(1)} \supseteq \ldots
$$

by setting $G_{(i+1)}=\left[G, G_{(i)}\right]$ for each $i \geq 0$. Recall that $G$ is nilpotent if $G_{(i)}=1$ for $i$ sufficiently large.

A group $G$ is said to be polycyclic if it has a finite normal series

$$
G=G_{0} \supseteq G_{1} \supseteq \ldots \supseteq G_{l}=1
$$

$\left(G_{i} \triangleleft G\right.$ for $\left.i=1, \ldots, l\right)$ for which each factor $G_{i-1} / G_{i}$ is cyclic (finite or 
infinite). Then the number $h(G)$ of infinite cyclic terms occurring in a series as above is an invariant of $G$ (independent of the series) known as the Hirsch number of $G$ (see [R, 5.4.13]).

We call a group $G$ a poly-ZZ-group if it has a finite normal series with factors $\mathbb{Z}$.

Since every finitely generated nilpotent group is polycyclic (see for example [Ru, 15.4, p. 92]), for finitely generated groups we have the following inclusions:

$0 \subseteq$ abelian groups $\subseteq$ nilpotent groups $\subseteq$ polycyclic groups.

A group $G$ is polycyclic-by-finite if it is an extension of a polycyclic group by a finite group, i.e., there exists a polycyclic group $H \triangleleft G$ such that $G / H$ is finite.

A homomorphism $f: G \rightarrow H$ of groups is an $r$-homomorphism if there exists a homomorphism $g: H \rightarrow G$ such that $f g=\operatorname{id}_{H}$. Then $H$ is an $r$-image of $G$ (see [B3, Ch. 2]).

Recall that a group $G$ is called Hopfian (see [Ba]) if every epimorphism $f: G \rightarrow G$ is an automorphism (equivalently, $N=1$ is the only normal subgroup for which $G / N \cong G$ ).

Similarly, a module $M$ is called Hopfian (see [Hi]) if every epimorphism $f: M \rightarrow M$ is an isomorphism (equivalently, $M$ is not isomorphic to a proper quotient of itself).

Let $R$ be a ring. Recall that a right $R$-module $M$ is said to be noetherian if every $R$-submodule of $M$ is finitely generated (see [La, Ch. VI.1]). We call a ring $R$ noetherian if it is a noetherian module as a left module over itself.

3. Main results. We start by showing that every nilpotent polyhedron has finite depth.

Definition 1. A $C W$-complex $X$ is said to be nilpotent if $X$ is connected, $\pi_{1}(X)$ is nilpotent and, for every integer $i \geq 2, \pi_{1}(X)$ acts nilpotently on $\pi_{i}(X)$ (see $\left.[\mathrm{HMR}]\right)$.

Recall that an action of a group $G$ on an abelian group $M$ is nilpotent if there exists a finite composition series

$$
0=M_{0} \subseteq M_{1} \subseteq \ldots \subseteq M_{n}=M
$$

such that $G$ acts trivially on $M_{j} / M_{j-1}$ for $1 \leq j \leq n$.

Definition 2. A $C W$-complex $X$ is called simple if $\pi_{1}(X)$ is abelian and acts trivially on all higher homotopy groups $\pi_{i}(X)(i \geq 2)$.

Examples of nilpotent polyhedra are, clearly, all the simply-connected finite $C W$-complexes and simple finite $C W$-complexes (hence finite $\mathrm{H}$-spaces), and also Eilenberg-MacLane $C W$-spaces $K(G, 1)$ for every finitely gen- 
erated nilpotent torsion free group $G$-it is known that they have homotopy types of finite $C W$-complexes (see for instance [Br, Ch. 8, Theorem (7.1) and Ch. 6]). On the other hand, an example of a non-nilpotent finite $C W$ complex with abelian fundamental group is the projective plane $\mathbb{R} P^{2}$ (and all the projective spaces $\mathbb{R} P^{n}$ for even $n$ ).

We prove the following:

Theorem 1. Let $P$ be a nilpotent polyhedron. Then $D(P)$ is finite.

Proof. Observe that any $C W$-complex which is homotopy dominated by a nilpotent $C W$-complex $P$ is also nilpotent. Firstly, any $r$-image of a nilpotent group is also nilpotent since it is known that a homeomorphic image of a nilpotent group is nilpotent (see [R, 5.1.4]).

Suppose that for a finitely generated abelian group $M$ there exists a finite composition series

$$
0=M_{0} \subseteq M_{1} \subseteq \ldots \subseteq M_{n}=M
$$

such that $G$ acts trivially on each $M_{j} / M_{j-1}(1 \leq j \leq n)$. Let $H$ be a retract of $G$, and let $r: M \rightarrow N$ be an $r$-homomorphism. Then there exists a composition series

$$
0=N_{0} \subseteq N_{1} \subseteq \ldots \subseteq N_{n}=N
$$

in which $H$ acts trivially on $N_{j} / N_{j-1}$ for $1 \leq j \leq n$. It suffices to take $N_{j}=r\left(M_{j}\right)$ for $1 \leq j \leq n$.

A theorem of Dror [Dr] (see [Ge, Theorem, p. 259]) states that if $f: X \rightarrow Y$ is a map of connected $C W$-complexes which induces an isomorphism on integral homology groups, and $X$ and $Y$ are nilpotent spaces, then $f$ is a homotopy equivalence.

By the well-known structure theorem for finitely generated abelian groups ([La, Ch. I.10]), any such group has only finitely many $r$-images up to isomorphism. Moreover, an $r$-homomorphism between isomorphic finitely generated abelian groups is an isomorphism. This follows for example from the fact that if $G$ and $H$ are abelian groups and $\varphi: G \rightarrow H$ is an $r$ homomorphism, then $G \cong H \times \operatorname{ker} \varphi$ ([B3, Ch. II, Theorem 1.6]; or [Mo, (1.3) p. 67]), and from the structure of finitely generated abelian groups again.

Thus, for each nilpotent polyhedron $P, D(P)$ is finite.

Answering a question of K. Borsuk [B1, problem (8)] we now show that there exist polyhedra, even with nilpotent fundamental group, with infinite capacity and finite depth. For every non-abelian poly-ZZ-group $G$ and integer $n \geq 3$, there exists an $n$-dimensional polyhedron $P$ with fundamental group $\pi_{1}(P) \cong G$ with this property. 
Definition 3. For a group $G$ and integer $n \geq 2$, by a $(G, n)$-complex we mean a finite, connected $C W$-complex $X$ of dimension $\leq n$ with $\pi_{1}(X) \cong G$ and $\pi_{i}(X)=0$ for $1<i<n$.

Definition 4. Recall that a $\mathbb{Z} G$-module $M$ is finitely generated projective if it is a direct summand of a finitely generated free $\mathbb{Z} G$-module $(\mathbb{Z} G)^{(n)}$, where $(\mathbb{Z} G)^{(n)}$ denotes the direct sum of $n$ copies of $\mathbb{Z} G$, and $n$ is an integer.

Two finitely generated projective $\mathbb{Z} G$-modules $M$ and $N$ are called stably equivalent if there exist two finitely generated free $\mathbb{Z} G$-modules $(\mathbb{Z} G)^{(k)}$ and $(\mathbb{Z} G)^{(l)}$ such that

$$
M \oplus(\mathbb{Z} G)^{(k)} \cong N \oplus(\mathbb{Z} G)^{(l)},
$$

where $k$ and $l$ are integers.

Under the operation of direct sum the stable equivalence classes of finitely generated projective modules form a group denoted by $\widetilde{K}^{0}(\mathbb{Z} G$ ) (see for example [Wa]). A finitely generated projective $\mathbb{Z} G$-module $M$ is called stably free if it represents 0 in $\widetilde{K}^{0}(\mathbb{Z} G)$.

Let us begin with the following:

LEMMA 1. Let $G$ be a finitely presented group such that the integral group ring $\mathbb{Z} G$ is noetherian, and let $n \geq 3$. Then for every finitely generated stably free $\mathbb{Z} G$-module $Q$ there exists a $(G, n)$-complex $X$ with $\pi_{n}(X) \cong Q$.

Proof. We first build by induction a finite $C W$-complex $K$ of dimension $n+1$ such that $\pi_{1}(K) \cong G, \pi_{i}(K)=0$ for $2 \leq i \leq n-1$ and $\pi_{n}(K) \cong Q$.

We begin by constructing in the well-known way a finite 2 -dimensional $C W$-complex $K_{2}$ with $\pi_{1}\left(K_{2}\right) \cong G$.

Suppose that we have built a finite $C W$-complex $K_{r}$ of dimension $r \geq 2$ such that $\pi_{1}\left(K_{r}\right) \cong G$ and $\pi_{i}\left(K_{r}\right) \cong 0$ for $2 \leq i \leq r-1$. Observe that the $\mathbb{Z} G$-module $\pi_{r}\left(K_{r}\right)$ is finitely generated.

To see this, recall that for every finite $C W$-complex $L$, the $k$-chains in the cellular complex of the universal cover $\widetilde{L}, C_{k}(\widetilde{L})$, have the structure of a finitely generated module over $\mathbb{Z} \pi_{1}(L)$ with a basis corresponding to the $k$-cells of $L$ (J. H. C. Whitehead [Wh1], or for example [C, Ch. 2, p. 28]).

Thus $C_{r}\left(\widetilde{K}_{r}\right)$ is a finitely generated $\mathbb{Z} G$-module.

Observe that $\pi_{r}\left(K_{r}\right) \cong \pi_{r}\left(\widetilde{K}_{r}\right) \cong H_{r}\left(\widetilde{K}_{r}\right)$ as $\mathbb{Z} G$-modules.

Since $\mathbb{Z} G$ is a noetherian ring, every finitely generated $\mathbb{Z} G$-module is noetherian (see $[\mathrm{La}, \mathrm{Ch} . \mathrm{VI}]$ ) and so any submodule of a finitely generated $\mathbb{Z} G$-module is also finitely generated. Since $Z_{r}\left(\widetilde{K}_{r}\right)$ is a submodule of $C_{r}\left(\widetilde{K}_{r}\right)$, it is a finitely generated $\mathbb{Z} G$-module. Thus $H_{r}\left(\widetilde{K}_{r}\right)$, a quotient module of $Z_{r}\left(\widetilde{K}_{r}\right)$, is also a finitely generated $\mathbb{Z} G$-module. Therefore $\pi_{r}\left(K_{r}\right)$ is a finitely generated module over $\mathbb{Z} G$. 
Then we can attach finitely many $(r+1)$-cells to $K_{r}$ to kill the generators of $\pi_{r}\left(K_{r}\right)$ and obtain $K_{r+1}$ with $\operatorname{dim} K_{r+1}=r+1, \pi_{1}\left(K_{r}\right) \cong G$, and $\pi_{i}\left(K_{r}\right)=0$ for $2 \leq i \leq r$.

We stop the construction at dimension $n$, obtaining a finite $C W$-complex $K_{n}$ with $\operatorname{dim} K_{n}=n, \pi_{1}\left(K_{n}\right) \cong G$, and $\pi_{i}\left(K_{n}\right)=0$ for $2 \leq i \leq n-1$.

Let $l$ be an integer such that $Q \oplus(\mathbb{Z} G)^{(k)} \cong(\mathbb{Z} G)^{(l)}$ for some integer $k$. Take a one-point union $K$ of $K_{n}$ and $l n$-spheres $S^{n}, K=K_{n} \vee \bigvee_{l} S^{n}$.

It is well known that, for any $G$ and any $(G, m)$-complex $L$, we have an isomorphism of $\mathbb{Z} G$-modules $\pi_{m}\left(L \vee S^{m}\right) \cong \pi_{m}(L) \oplus \pi_{m}\left(S^{m}\right)$ (folklore; see for example [CS, Appendix]).

Thus $\pi_{n}(K) \cong \pi_{n}\left(K_{n}\right) \oplus \mathbb{Z} G^{(l)}$. Now we can attach to $K_{n}$ a finite number of $(n+1)$-cells to kill a finite generating set of the $\mathbb{Z} G$-module $\pi_{n}\left(K_{n}\right)$ and then attach $l(n+1)$-cells to the wedge $\bigvee_{l} S^{n}$ by the maps corresponding to the homomorphism $(\mathbb{Z} G)^{(l)} \cong Q \oplus(\mathbb{Z} G)^{(k)} \stackrel{p}{\rightarrow}(\mathbb{Z} G)^{(k)}$ on the generators, where $p$ is the projection on the summand $(\mathbb{Z} G)^{(k)}$. This way we obtain a finite $C W$-complex $X$ with $\pi_{1}(X) \cong G, \pi_{i}(X)=0$ for $2 \leq i \leq n-1$, $\pi_{n}(X) \cong Q$, and $\operatorname{dim} X=n+1$.

We will now modify $X$ to an $n$-dimensional finite $C W$-complex $X^{\prime}$ with the same homotopy groups in dimensions $1, \ldots, n$ by the method used in [Dy] (cf. [Dy, Proposition 1.4]). Consider the cellular chain complex of the universal cover $\widetilde{X}$ of $X$,

$$
\mathcal{C}: C_{n+1}(\tilde{X}) \stackrel{\partial_{n+1}}{\longrightarrow} C_{n}(\tilde{X}) \stackrel{\partial_{n}}{\longrightarrow} C_{n-1}(\tilde{X}) \stackrel{\partial_{n-1}}{\longrightarrow} C_{n-2}(\widetilde{X}) \stackrel{\partial_{n-2}}{\longrightarrow} \ldots \stackrel{\partial_{1}}{\longrightarrow} C_{0}(\widetilde{X}) .
$$

Since $\pi_{n}(X) \cong C_{n}(\tilde{X}) / B_{n}(\tilde{X}) \cong Q$, we have $C_{n}(\tilde{X}) / B_{n}(\tilde{X}) \oplus(\mathbb{Z} G)^{(k)} \cong$ $(\mathbb{Z} G)^{(l)}$ for some integers $k$ and $l$. It is easy to see that the following chain complex, in which $\partial_{n}^{\prime}=\partial_{n} \oplus \mathrm{id}, \partial_{n-1}^{\prime}=\partial_{n-1} \oplus 0$ and $\partial_{i}^{\prime}=\partial_{i}$ for $i \leq n-2$, is also a complex of free, finitely generated $\mathbb{Z} G$-modules:

$$
\mathcal{C}^{\prime}: C_{n}(\widetilde{X}) / B_{n}(\widetilde{X}) \oplus(\mathbb{Z} G)^{(k)} \stackrel{\partial_{n}^{\prime}}{\longrightarrow} C_{n-1}(\widetilde{X}) \oplus(\mathbb{Z} G)^{(k)} \stackrel{\partial_{n-1}^{\prime}}{\longrightarrow} C_{n-2}(\widetilde{X}) \stackrel{\partial_{n-2}^{\prime}}{\longrightarrow} \ldots
$$

Now let us change $X$ into an $n$-dimensional finite $C W$-complex as follows. Take

$$
X^{\prime}=\left(X^{(n-1)} \vee \bigvee_{k} S^{n-1}\right) \cup \bigcup_{i=1}^{l} e_{i}^{n},
$$

with the $n$-cells attached to $X^{(n-1)} \vee \bigvee_{k} S^{n-1}$ by the maps corresponding to the homomorphism $\partial_{n}^{\prime}:(\mathbb{Z} G)^{(l)} \rightarrow C_{n-1}(\widetilde{X}) \oplus(\mathbb{Z} G)^{(k)}$ on the generators of $(\mathbb{Z} G)^{(l)}$. Then $\pi_{n}\left(X^{\prime}\right) \cong H_{n}\left(\widetilde{X^{\prime}}\right)=\operatorname{ker} \partial_{n}^{\prime} /$ im $\partial_{n+1}^{\prime} \cong \pi_{n}(X)$. Thus the proof is complete.

COROllary 1. Let $G$ be a polycyclic-by-finite group. Then for every projective, finitely generated, stably free $\mathbb{Z} G$-module $Q$ and $n \geq 3$ there exists a $(G, n)$-complex $X$ with $\pi_{n}(X) \cong Q$. 
Proof. This follows from Lemma 1. It is known that every poly-(cyclic or finite) group is finitely presented (for this result of P. Hall see, for example, [R, Lemma 2.2 .4 , p. 53 , and p. 54]). Hence $G$ is a finitely presented group. Let us remark that a group is poly-(cyclic or finite) if and only if it is polycyclic-by-finite (see for example [S, Proposition 2, p. 2]).

If $G$ is a polycyclic-by-finite group, then by another result of Hall, $\mathbb{Z} G$ is a noetherian ring. Indeed, Hall proved that if $G$ is a finite extension of a polycyclic group and $R$ is a right noetherian ring with identity, then the group ring $R G$ is right noetherian (see [R, 15.3.3, p. 446]).

In the case of poly-ZZ-groups, the corollary may be formulated as follows:

Corollary 2. Let $G$ be a poly-ZZ-group. Then for every projective, finitely generated $\mathbb{Z} G$-module $Q$ and $n \geq 3$ there exists a $(G, n)$-complex $X$ with $\pi_{n}(X) \cong Q$.

Proof. This is a consequence of Corollary 1 . Note that for every poly$\mathbb{Z}$-group $G, \widetilde{K}^{0}(\mathbb{Z} G)=0$ (see $[\mathrm{Wd}],[\mathrm{Q}]$ ), thus every projective, finitely generated $\mathbb{Z} G$-module is stably free.

In what follows we will also use:

Lemma 2. Let $G$ be a poly-Z-Zroup. There exists an integer $k$ such that any descending sequence of subgroups

$$
G=G_{0} \supseteq G_{1} \supseteq \ldots
$$

with retractions $r_{i}: G_{i-1} \rightarrow G_{i}$ for $i=1,2 \ldots$ contains no more than $k$ distinct subgroups.

Proof. Note that if $h(G)$ is the Hirsch number of a poly- $\mathbb{Z}$-group $G$, $H \subseteq G$ and $N \triangleleft G$ is such that $H=G / N$, then $h(G)=h(N)+h(H)$ (see e.g. [S, Ex. 8, p. 16]).

Let $M_{i}$ be the kernel of the retraction $r_{i}: G_{i-1} \rightarrow G_{i}$. Then

$$
h\left(G_{i-1}\right)=h\left(M_{i}\right)+h\left(G_{i}\right)
$$

for $i=1,2, \ldots$, thus

$$
h(G)=h\left(M_{1}\right)+\ldots+h\left(M_{i}\right)+h\left(G_{i}\right) .
$$

Hence there exists an integer $k$ such that $h\left(M_{i}\right)=0$ for all $i$ except no more than $k$. Then $M_{i}=0$ for such $i$. Indeed, being a subgroup of a poly-ZZZ-group, $M_{i}$ is a poly-ZZZ-group, and clearly, if a poly-ZZ-group has Hirsch number 0 , then it is trivial.

Therefore $G_{i}=G_{i-1}$ for those $i$.

Thus, every descending sequence of subgroups of $G$ contains no more than $k$ different groups, where $k-1=h(G)$ is the Hirsch number of $G$. This ends the proof. 
The following theorem was proved by J. C. H. Whitehead in 1949:

Theorem ([Wh2, Theorem 14]; see also [Wh1]). Suppose that $X$ and $Y$ are $(G, n)$-complexes. Then there exist integers $a_{X}$ and $a_{Y}$ such that

$$
X \vee \bigvee_{a_{X}} S^{n} \simeq Y \vee \bigvee_{a_{Y}} S^{n}
$$

where $\bigvee_{k} S^{n}$, for an integer $k$, denotes the wedge of $k$ spheres $S^{n}$.

Definition 5 (see, for example, [Dy, p. 249]). By a homotopy tree $\operatorname{HT}(G, n)$ we mean a directed tree whose vertices are all the homotopy types of $(G, n)$-complexes and where the homotopy type of $X$ is joined by an edge to the homotopy type of $X \vee S^{n}$.

The tree is partitioned into levels by the Euler-Poincaré characteristic, i.e. level $(X)=(-1)^{n} \chi(X)-\chi_{\min }$, where $\chi_{\min }$ is the minimum of $(-1)^{n} \chi(Y)$ over all the $(G, n)$-complexes $Y$.

Let us prove the following:

Lemma 3. Let $G$ be a finitely presented group such that $G$ is Hopfian and the integral group ring $\mathbb{Z} G$ is noetherian. Suppose that $X_{k}$ and $X_{l}$ are two $(G, n)$-complexes, where $n \geq 2$, and $X_{k} \not 千 X_{l}$. Then $X_{k} \leq X_{l}$ implies that level $\left(X_{k}\right)<\operatorname{level}\left(X_{l}\right)$.

Proof. We first show that if $X_{l} \geq X_{k}$ and $X_{k} \nsucceq X_{l}$, then there exists a non-trivial $\mathbb{Z} G$-module $S$ such that

$$
\pi_{n}\left(X_{k}\right) \oplus S \cong \pi_{n}\left(X_{l}\right) .
$$

To see this, observe that the domination $X_{l} \geq X_{k}$ induces an isomorphism of the fundamental groups $\pi_{1}\left(X_{l}\right) \rightarrow \pi_{1}\left(X_{k}\right) \cong \pi_{1}\left(X_{l}\right)$. This follows from the fact that $\pi_{1}\left(X_{l}\right) \cong G$ is Hopfian. Indeed, every $r$-homomorphism between isomorphic Hopfian groups is an isomorphism.

Recall that $\pi_{i}\left(X_{k}\right) \cong \pi_{i}\left(X_{l}\right)=0$ for $2 \leq i \leq n-1$. Suppose now that the domination $X_{l} \geq X_{k}$ induces an isomorphism $\pi_{n}\left(X_{l}\right) \rightarrow \pi_{n}\left(X_{k}\right)$. Then, by the Whitehead theorem, $X_{k}$ and $X_{l}$ should be homotopy equivalent, which is not the case. Thus $X_{l} \geq X_{k}$ and $X_{k} \nsucceq X_{l}$ implies that there exists a non-trivial $\mathbb{Z} G$-module $S$ such that $\pi_{n}\left(X_{k}\right) \oplus S \cong \pi_{n}\left(X_{l}\right)$.

On the other hand, by the Whitehead theorem on trees, there exist integers $a$ and $b$ such that

$$
X_{k} \vee \bigvee_{a} S^{n} \simeq X_{l} \vee \bigvee_{b} S^{n}
$$

Thus

$$
\pi_{n}\left(X_{k}\right) \oplus(\mathbb{Z} G)^{(a)} \cong \pi_{n}\left(X_{l}\right) \oplus(\mathbb{Z} G)^{(b)}
$$

as $\mathbb{Z} G$-modules (for a $(G, n)$-complex $X$, we have an isomorphism of $\mathbb{Z} G$ modules $\pi_{n}\left(X \vee S^{n}\right) \cong \pi_{n}(X) \oplus \pi_{n}\left(S^{n}\right)$ - see the proof of Lemma 1$)$. 
Suppose that $b \geq a$. Then, for the $\mathbb{Z} G$-module $T=\pi_{n}\left(X_{k}\right) \oplus(\mathbb{Z} G)^{(a)}$, we have $T \oplus S \oplus(\mathbb{Z} G)^{(b-a)} \cong T$, where $b-a \geq 0$. Thus $T$ is isomorphic to a proper factor module of itself. But this contradicts the fact that the $\mathbb{Z} G$-module $T$ is Hopfian.

That $T$ is Hopfian follows from the facts that if the integral group ring $\mathbb{Z} G$ is noetherian, then any finitely generated $\mathbb{Z} G$-module is noetherian ([La, Ch. VI]; compare the proof of Lemma 1), and that if a finitely generated module is right noetherian, then it is Hopfian (see, for example, [Hi, Proposition 6]). Observe that $T$ is a finitely generated module over $\mathbb{Z} G$ because $\pi_{n}\left(X_{k}\right)$ is a finitely generated module over $\mathbb{Z} G$ (by the arguments as in the proof of Lemma 1).

Thus we have shown that $b<a$. This means that level $\left(X_{k}\right)<\operatorname{level}\left(X_{l}\right)$ and finishes the proof.

Corollary 3. Let $G$ be a polycyclic-by-finite group. Suppose that $X_{k}$ and $X_{l}$ are two $(G, n)$-complexes, where $n \geq 2$, and $X_{k} \not X_{l}$. Then $X_{k} \leq X_{l}$ implies that level $\left(X_{k}\right)<\operatorname{level}\left(X_{l}\right)$.

Proof. In fact, Hirsch proved that every polycyclic-by-finite group is residually finite, i.e. its subgroups of finite index have trivial intersection (see [R, 5.4.17] or [S, Theorem 1, p. 17]). It is also known that if a finitely generated group $G$ is residually finite, then it is Hopfian (see [Ma], [KS]). Moreover, if $G$ is polycyclic-by-finite, then the integral group ring $\mathbb{Z} G$ is noetherian (see the proof of Corollary 1).

Thus the assertion follows from Lemma 3.

Definition 6. $C W$-complexes $X$ and $Y$ have the same $n$-type if there exists a map $f: X^{(n+1)} \rightarrow Y^{(n+1)}$ such that $f_{i *}: \pi_{i}\left(X^{(n+1)}\right) \rightarrow \pi_{i}\left(Y^{(n+1)}\right)$ is an isomorphism for all $1 \leq i \leq n$ (see [Wh1]).

Note that any two $(G, n)$-complexes have the same $(n-1)$-type.

We now prove our main results. The next theorem gives an answer to the question of $\mathrm{K}$. Borsuk mentioned in the introduction:

TheOREM 2. For every non-abelian poly-ZZ-group $G$ and an integer $n \geq 3$, there exists a polyhedron $P$ such that $\pi_{1}(P) \cong G, \operatorname{dim} P=n, C(P)$ is infinite, but $D(P)$ is finite.

Proof. In the first step we will obtain a polyhedron $P$ with $\pi_{1}(P) \cong G$ and $\operatorname{dim} P=n$, homotopy dominating infinitely many polyhedra of different homotopy types.

The results of Artamonow [Ar] imply that for every non-abelian poly- $\mathbb{Z}$ group $G$ there exist infinitely many non-isomorphic modules over $\mathbb{Z} G$, say $M_{i}, i=1,2, \ldots$ (of rank 1 ), which are projective, finitely generated and (hence) stably free. Precisely, we have $M_{i} \oplus \mathbb{Z} G \cong \mathbb{Z} G \oplus \mathbb{Z} G$. 
By Corollary 2, for each $i$ and $n \geq 3$, there is a finite $(G, n)$-complex $X_{i}$ with $\pi_{n}\left(X_{i}\right) \cong M_{i}$. Clearly the $X_{i}$ have different homotopy types. But there exists an integer $d$ such that $X_{i} \vee \bigvee_{d} S^{n} \simeq P$ for some polyhedron $P$.

We can deduce this, for example, from the following fact (see [K2, Lemma 1.1, p. 273]) whose proof is similar to the proof of the Whitehead theorem on trees ([Wh2, Theorem 14, p. 49]): Let $Z_{i}$, for $i=1,2, \ldots$, be finite $C W$-complexes with $\operatorname{dim} Z_{i}=n$ such that the $(n-1)$-types of all $Z_{i}$ are equal. Suppose that for each $r$ and all $i$, the number of $r$-cells in $Z_{i}$ is equal to $d_{r}$, where $d_{r}$ is some integer. Then there exists an integer $d$ such that the $Z_{i} \vee \bigvee_{d} S^{n}$ are all homotopy equivalent.

Observe that in our situation, by the construction from the proof of Lemma 1, each $X_{i}$ has a fixed number of cells in each dimension. Since on ANRs shape and homotopy theories coincide, for such a $P$ we have immediately $C(P)=\aleph_{0}$.

We now show that $D(P)$ is finite. According to the remark in the introduction, in the definition of the depth of a polyhedron one can always consider homotopy dominations over $C W$-complexes (not necessarily finite).

In the case where $\pi_{1}(P) \cong G$ is a poly-ZZ-group, we may assume these $C W$-complexes to be finite. This follows from the known result of Wall (or directly from its shape analogue): if $X$ is homotopy dominated by a polyhedron $P$ with $\operatorname{dim} P=m \geq 2$ and $\widetilde{K}^{0}\left(\mathbb{Z} \pi_{1}(X)\right)=0$, then $X$ is homotopy equivalent to a polyhedron of dimension $\leq \max (m, 3)$ (see $[\mathrm{Wa}$, Theorem $\mathrm{F}$, p. 66] or [EG, Theorem 1.1], respectively). Recall that $\widetilde{K}^{0}(\mathbb{Z} G)=0$ for every poly-ZZ-group $G$ and all of its subgroups, which are also poly-ZZ-groups.

Thus, every $X \leq P$, where $P$ satisfies the assumptions of the theorem, is homotopy equivalent to a polyhedron of dimension $\leq n$.

Since $\pi_{1}(P) \cong G$ is a poly-ZZ-group, by Lemma 2 , there exists an integer $s$ such that for each sequence of polyhedra

$$
P=X_{0} \geq X_{1} \geq \ldots \geq X_{j-1} \geq X_{j} \geq \ldots,
$$

there are at most $s$ non-isomorphic groups among $\pi_{1}\left(X_{j}\right)$. Hence every $X_{j}$ in such a sequence is an $(H, n)$-complex, where $H$ is one of at most $s r$-images of $G$.

Furthermore, by Corollary 3, if $X_{k}$ and $X_{l}$ are two polyhedra from the tree of the same poly-ZZ-group $H$, then $X_{k} \leq X_{l}$ and $X_{k} \nsucceq X_{l}$ implies that $\operatorname{level}\left(X_{l}\right)<\operatorname{level}\left(X_{k}\right)$.

Now it is easy to see that $D(P)$ is finite and the proof is finished.

EXAMPLE. The first example of a poly-ZZ-group $G$ such that there exist infinitely many non-isomorphic projective modules over $\mathbb{Z} G$ was the group with presentation

$$
G=\left\langle r, p, q \mid p q=q p, q=r^{-1} p r, r^{-1} q r=q p^{-1}, r p r^{-1}=q^{-1} p, r q r^{-1}=p\right\rangle
$$


(see $[\mathrm{BeDu}]$ ). It is known that $G=T / T^{(2)}$, where $T$ is the trefoil knot group, i.e. the group with presentation

$$
T=\left\langle a, b \mid a^{2}=b^{3}\right\rangle,
$$

and $T^{(2)}=\left(T^{\prime}\right)^{\prime}\left(\right.$ where $\left.G^{\prime}=[G, G]\right)$.

Corollary 4. For every non-abelian nilpotent torsion-free finitely generated group $G$ there exists a polyhedron $P$ with $\pi_{1}(P) \cong G$ such that $C(P)$ is infinite, but $D(P)$ is finite.

Proof. Every finitely generated, nilpotent torsion-free group is a poly$\mathbb{Z}$-group (see for example [R, Theorem 5.2.20, p. 133]). Thus the statement follows from Theorem 2 .

There are many examples of finitely generated nilpotent torsion-free nonabelian groups, thus we obtain:

COROLlary 5. There exists a polyhedron $P$ with nilpotent fundamental group such that $C(P)$ is infinite, but $D(P)$ is finite.

Now let us state the following questions:

Question 1. Is it true that for each polyhedron $P$ such that $\pi_{1}(P)$ is abelian, $C(P)$ is finite?

Question 2. Is it true that for each polyhedron $P$ such that $\pi_{1}(P)$ is a poly-ZZ-group (or nilpotent group), $D(P)$ is finite?

It seems to the author that the answer to both questions is affirmative.

3. Final remarks. In the case of nilpotent torsion-free finitely generated groups, instead of poly-ZZ-groups, the proof of Lemma 2 can be neatly simplified. We show below how one can prove it "topologically".

Proposition. For every finitely generated nilpotent torsion-free group $G$, there exists an integer $k$ such that any descending sequence of subgroups

$$
G=G_{0} \supseteq G_{1} \supseteq \ldots
$$

with retractions $r_{i}: G_{i-1} \rightarrow G_{i}$ for $i=1,2, \ldots$ contains no more than $k$ distinct subgroups.

Proof. Recall that for every finitely generated torsion-free nilpotent group $G$, there exists a finite $C W$-complex $K(G, 1)$ of dimension equal to the Hirsch number of $G$ (see for example [Br, Ch. 8, Theorem (7.1) and Ch. 6]). Every $r$-image of a finitely generated group is clearly finitely generated. Thus every $r$-image of a finitely generated nilpotent torsion-free group is also a group of this kind. So let us associate with each retract $H$ of $G$ a finite $C W$-complex $K(H, 1)$. Obviously these spaces are all nilpotent. Recall that for every homomorphism $\varphi: \pi \rightarrow \varrho$ of groups, there exists a unique 
(up to homotopy) pointed map $f: K(\pi, 1) \rightarrow K(\varrho, 1)$ such that $\pi_{1}(f)=\varphi$. Since $H$ is a retract of $G$ it follows that $K(H, 1)$ is homotopy dominated by $K(G, 1)$.

By Theorem 1 , the depth of $K(G, 1)$ is finite. This means that every descending sequence of subgroups

$$
G=G_{0} \supseteq G_{1} \supseteq \ldots
$$

with retractions $r_{i}: G_{i-1} \rightarrow G_{i}$ for $i=1,2, \ldots$ contains no more than $k$ different groups up to isomorphism, hence no more than $k$ different groups. This ends the proof.

REMARK. The author expects that Lemma 2 could be proven topologically even for poly-ZZ-groups, perhaps in a more complicated way.

Acknowledgments. The author would like to thank Prof. Stanisław Spież for valuable conversations and remarks.

\section{References}

[Ar] V. A. Artamonov, Projective non-free modules over group rings of solvable groups, Mat. Sb. 116 (1981), 232-244 (in Russian).

[Ba] G. Baumslag, Topics in Abelian Groups, ed. by J. Irwin and E. A. Walker, 1943.

[BeDu] P. H. Berridge and M. J. Dunwoody, Non-free projective modules for torsion-free groups, J. London Math. Soc. (2) 19 (1979), 433-436.

[B1] K. Borsuk, Some problems in the theory of shape of compacta, Russian Math. Surveys 34 (1979), no. 6, 24-26.

[B2] -, Theory of Shape, Monografie Mat. 59, Polish Sci. Publ., Warszawa, 1975.

[B3] -, Theory of Retracts, Monografie Mat. 44, Polish Sci. Publ. 44, Warszawa, 1967.

[Br] K. Brown, Cohomology of Groups, Springer, Berlin, 1982.

[CS] W. H. Cockroft and R. G. Swan, On the homotopy types of certain two-dimensional complexes, Proc. London Math. Soc. (3) 11 (1964), 194-202.

[C] M. Cohen, A Course in Simple Homotopy Theory, Grad. Texts in Math. 10, Springer, Berlin, 1970.

[Dr] E. Dror, A generalization of the Whitehead theorem, in: Symposium on Algebraic Topology, Lecture Notes in Math. 249, Springer, Berlin, 1971, 13-22.

[D] J. Dydak, Pointed and unpointed shape and pro-homotopy, Fund. Math. 107 (1980), 58-69.

[DKN] J. Dydak, A. Kadlof and S. Nowak, Open Problems in Shape Theory, mimeographed notes, University of Warsaw, 1981.

[DS] J. Dydak and J. Segal, Shape Theory: An Introduction, Lecture Notes in Math. 688, Springer, Berlin, 1978.

[Dy] M. N. Dyer, Homotopy classification of $(\pi, m)$-complexes, J. Pure Appl. Algebra 7 (1976), 249-282.

[EG] D. A. Edwards and R. Geoghegan, Shapes of complexes, ends of manifolds, homotopy limits and the Wall obstruction, Ann. of Math. (2) 101 (1975), 521535; Correction, ibid. 104 (1976), 389. 
[Ge] S. M. Gersten, The Whitehead theorem for nilpotent spaces, Proc. Amer. Math. Soc. 47 (1975), 259-260.

[HaHe1] H. M. Hastings and A. Heller, Homotopy idempotents on finite-dimensional complexes split, Proc. Amer. Math. Soc. 85 (1982), 619-622.

[HaHe2] - - - Splitting homotopy idempotents, in: Shape Theory and Geometric Topology (Dubrovnik, 1981), Lecture Notes in Math. 870, Springer, Berlin, 1981, 23-36.

[HMR] P. J. Hilton, G. Mislin and J. Roitberg, Localization of Nilpotent Groups and Spaces, North-Holland, 1975.

[Hi] V. A. Hiremath, Hopfian rings and Hopfian modules, Indian J. Pure Appl. Math. 17 (1986), 895-900.

[Ho] W. Holsztyński, A remark on homotopy and category domination, Michigan Math. J. 18 (1971), 409.

[KS] A. Karras and D. Solitar, On free products, Proc. Amer. Math. Soc. 9 (1958), $217-221$.

[K] D. Kołodziejczyk, On shape dominations for some classes of compacta, Ph.D. thesis, Univ. of Warsaw, 1993.

[K1] - On some problems of K. Borsuk concerning shape dominations, in: Baku International Topological Conference (Baku, 1987), હ̀lm, Baku, 1989, 243-246.

[K2] - Polyhedra dominating infinitely many different homotopy types, Bull. Polish Acad. Sci. Math. 44 (1996), 271-283.

[K3] - Polyhedra with finite fundamental group dominate only finitely many different homotopy types, Fund. Math., to appear.

[K4] - Simply-connected polyhedra dominate only finitely many different shapes, Topology Appl. 112 (2001), 289-295.

[K5] - There exists a polyhedron dominating infinitely many polyhedra of different homotopy types, Fund. Math. 151 (1996), 39-46.

[K6] - There exists a polyhedron with infinitely many left neighbors, Proc. Amer. Math. Soc. 129 (2001), 303-309.

[La] S. Lang, Algebra, Addison-Wesley, Reading, MA, 1965.

[Ma] A. I. Mal'tsev, On isomorphic representations of infinite groups by matrices, Mat. Sb. 8 (1940), 405-422 (in Russian).

[MS] S. Mardešić and J. Segal, Shape Theory. The Inverse System Approach, NorthHolland Math. Library 26, North-Holland, Amsterdam, 1982.

[M] M. Mather, Counting homotopy types of manifolds, Topology 4 (1965), 93-94.

[MP] M. A. Moron and F. R. Ruiz del Portal, Counting shape and homotopy types among fundamental absolute retracts: An elementary approach, Manuscripta Math. 79 (1993), 411-414.

[Mo] M. Moszyńska, On the homotopy classification of spaces, Fund. Math. 66 (1969), 65-83.

[Q] F. Quinn, Algebraic K-theory of poly-(finite or cyclic) groups, Bull. Amer. Math. Soc. 12 (1985), 221-226.

[R] D. J. S. Robinson, A Course in the Theory of Groups, Springer, 1982.

[Ru] J. Rutter, Spaces of Homotopy Self-Equivalences, Lecture Notes in Math. 1662, Springer, 1997.

[S] D. Segal, Polycyclic Groups, Cambridge Tracts in Math. 82, Cambridge Univ. Press, 1983.

[Wa] C. T. C. Wall, Finiteness conditions for CW-complexes, Ann. of Math. 81 (1965), 56-69. 
[Wd] F. Waldhausen, Whitehead groups of generalized free products, ibid. 108 (1978) 135-256.

[Wh1] J. H. C. Whitehead, Combinatorial homotopy I, II, Bull. Amer. Math. Soc. 55 (1949), 213-245, 453-496.

[Wh2] - Simple homotopy types, Amer. J. Math. 72 (1952), 1-57.

Faculty of Mathematics and Informational Sciences

Warsaw University of Technology

Pl. Politechniki 1

00-661 Warszawa, Poland

E-mail: dkolodz@mimuw.edu.pl

Institute of Mathematics

Polish Academy of Sciences

Śniadeckich 8

00-956 Warszawa, Poland

Received 31 July 2001;

in revised form 17 July 2003 